\title{
Decreased survival in patients with carcinoma of axillary tail versus upper outer quadrant breast cancers: a SEER population-based study
}

This article was published in the following Dove Press journal: Cancer Management and Research

\author{
Zong-Chao Gou ${ }^{1,2, *}$ \\ Xi-Yu Liu ${ }^{1,2, *}$ \\ Yi Xiao ${ }^{1,2}$ \\ Shen Zhao',2 \\ Yi-Zhou Jiang ${ }^{1,2}$ \\ Zhi-Ming Shaol-3 \\ 'Department of Breast Surgery, \\ Cancer Institute, Fudan University \\ Shanghai Cancer Center, Shanghai, \\ People's Republic of China; \\ 2Department of Oncology, Shanghai \\ Medical College, Fudan University, \\ Shanghai, People's Republic of China \\ ${ }^{3}$ Institutes of Biomedical Sciences, \\ Fudan University, Shanghai, People's \\ Republic of China
}

*These authors contributed equally to this work

Correspondence: Zhi-Ming Shao; Yi-Zhou Jiang

Department of Breast Surgery, Cancer Institute, Fudan University Shanghai

Cancer Center, 270 Dong-An Road,

Shanghai, 200032, People's Republic of

China

Tel +862164175590

Fax +862164434556

Email zhiminqshao@gmail.com;

yizhoujiang@fudan.edu.cn
Background: Carcinoma of the axillary tail of Spence (CATS) is a poorly studied type of breast cancer. The clinicopathological characteristics and prognostic features of CATS are unclear.

Methods: Using the Surveillance, Epidemiology, and End Results database, we identified 149,026 patients diagnosed with upper outer quadrant breast cancer (UOBC) $(n=146,343)$ or CATS ( $n=2,683)$. The median follow-up was 88 months. The primary and secondary outcomes were breast cancer-specific survival (BCSS) and overall survival. The survival outcomes of UOBC and CATS were compared using competing risks analysis, log-rank test, Cox proportional hazards regression model, and propensity score matching method. Multivariate logistic regression was utilized to present the relationship between CATS and lymph node (LN) metastasis. Results: CATS presented a higher grade, higher negative hormone receptor rate, and more positive nodal metastasis. The 10-year BCSS rate was worse for CATS than for UOBC $(85.1 \%$ vs $87.3 \%, P=0.001$ ). The multivariate Cox analysis showed a higher hazard ratio (HR) for CATS over UOBC (BCSS: $\mathrm{HR}=1.20, P=0.001$; overall survival: $\mathrm{HR}=1.11, P=0.019$ ). The difference in the BCSS was also observed in a 1:1 matched cohort (BCSS $P=0.019$ ). A subgroup analysis revealed the inferior outcomes of CATS in the metastatic LN subgroup and the hormone receptornegative subgroup. The multivariate logistic regression indicated that CATS is an independent contributing factor to LN metastasis.

Conclusion: CATS had distinct clinicopathological characteristics and was more likely associated with LN metastasis. Compared to UOBC, CATS had adverse impacts on BCSS.

Keywords: carcinoma of the axillary tail of Spence, upper outer quadrant breast cancer, breast cancer-specific survival, lymph node metastasis

\section{Introduction}

Breast cancer is one of the most common malignant tumors. In-depth research confirms various characteristics of independent prognostic factors, such as age, histologic type, grade, ER, PR, etc. ${ }^{1-3}$ Tumor location, as a basic and important variable, is acknowledged as a prognostic factor in cancer-specific survival. Past research of tumor location concludes that lower inner quadrant breast cancer has a worse outcome than upper outer quadrant cancer (UOBC) due to the undetected internal mammary chain. ${ }^{4-7}$ However, the effect of the tumor location of the axillary tail on survival has not been deeply explored yet.

The axillary tail of Spence (ATS) is variable in size, and it is recognized as a narrow extension of the upper outer quadrant breast (UOB) gland. ${ }^{8}$ The mammary gland of the ATS extends from the UOB and enters the axilla through a defect in the clavipectoral 
fascia. The ATS has a special anatomical position; that is, lymph nodes (LNs) are close to and can even be found in the axillary tail region. ${ }^{8}$

Carcinoma of the axillary tail of Spence (CATS) is one rare and poorly studied type of breast cancer. CATS accounts for $\sim 1 \%$ of breast cancers. ${ }^{7}$ CATS is one type of cancerous tumor located in the axillary fossa, which include occult breast cancer, ectopic breast cancer, and axillary metastasis from a nonbreast organ. ${ }^{9}$ Most studies about CATS are case reports. Ampil et $\mathrm{al}^{8}$ found that CATS had a tendency toward the more advanced stage and hormone receptor-negative neoplasms. Other researchers caution the recurrence of breast cancer in ATS after prophylactic mastectomy. ${ }^{9,10}$ However, it is challenging for case report studies to establish the prognosis of CATS, due to the absent or limited follow-up information as well as the small sample sizes. To date, the clinicopathological characteristics and prognostic features of CATS are still unclear. Moreover, due to the rarity of CATS, there is no specific guideline for it. One question that needs to be asked is whether CATS patients should receive the standard care management for anatomical breast cancer as a parallel disease stage. Therefore, more research is needed to unveil the characteristics of CATS.

In this study, we investigated the clinicopathological characteristics and prognostic features of CATS by utilizing one population-based database. UOBC was set as a reference due to three reasons. First, UOB is the most common site of breast cancer, accounting for 39\%-57\% of cases. ${ }^{7,11,12}$ Second, ATS and UOB are intimately related in terms of genesis and anatomical continuity. Previous research sometimes incorporated CATS in the UOBC category. ${ }^{7}$ Our research plan was to identify whether there is a clear clinicopathological and survival difference between CATS and UOBC. Third, when studying the impact of tumor site, researchers usually chose UOBC or outer quadrant breast cancer as a reference. ${ }^{4,11,13}$

\section{Methods}

\section{Database}

We used the SEER database (November 2016 submission), a National Cancer Institute-sponsored program, and obtained data from 18 population-based registries with the SEER*Stat software, version 8.3.4 (http://seer.cancer. gov/about/).

\section{Study population}

We derived a dataset of female breast cancer patients diagnosed from 1990 to 2012. The specific inclusion criteria were as follows: females aged between 18 and 79 years; American
Joint Committee on Cancer stages I-III; unilateral breast cancer; breast cancer as the first and only cancer diagnosis; cancer located in the ATS or UOB; diagnosis not obtained from a death certificate or autopsy; pathologic confirmation of breast cancer; known ER and PR statuses; known tumor size and LN status; known surgery type; and active follow-up.

Since 1990, patient ER and PR information has been recorded accessibly in the SEER database. The ER and PR results were combined as the hormone receptor (HoR) status. The National Comprehensive Cancer Network guidelines and some research suggest that breast cancers with at least $1 \%$ of cells positive for ER staining should be considered ER+. ${ }^{14-16}$ Therefore, patients with a borderline HoR status are defined as HoR+. The patients diagnosed after 2012 were excluded in order to ensure a follow-up time of 2 years at least. The flow diagram for the selection of the study cohort is presented in Figure S1. In total, 149,026 patients were included, including 2,683 CATS (1.80\%) and 146,343 UOBC patients $(98.20 \%)$.

\section{Ethics statement}

Our study was approved by an independent ethical committee/institutional review board at Fudan University Shanghai Cancer Center (Shanghai Cancer Center Ethical Committee). The data released by the Surveillance, Epidemiology, and End Results (SEER) database was publicly available and deidentified, and does not require patient's informed consent.

\section{Statistical analysis}

The demographic and clinicopathological characteristics were compared across CATS and UOBC using the Pearson $\chi^{2}$ test or Fisher's exact test, if appropriate. The primary and secondary outcomes of our study were breast cancerspecific survival (BCSS) and overall survival (OS), respectively. The BCSS was defined as the survival time from the diagnosis date of breast cancer to the date of death caused by breast cancer, and the OS was defined as the time from the diagnosis date of breast cancer to the date of death from any cause. Kaplan-Meier curves, competing risks analysis (for BCSS) and log-rank tests (for OS) were generated to determine differences in the survival analyses. Additionally, univariate and multivariate Cox proportional hazard models with hazard ratios (HRs) were applied to estimate the factors associated with the BCSS and OS. A 1:1 paired match, by the propensity score matching (PSM) method, was also carried out to balance the impact of the baseline. Subgroup analyses summarized the HR of CATS versus UOBC using a forest plot. Logistic regression was utilized to present the 
relationship between the breast cancer location and axillary LN metastasis.

Forest plots were generated with the function "forestplot" (R package: forestplot) in $\mathrm{R}$ version 3.2.2 (R Foundation for Statistical Computing, Vienna, Austria). All the other statistical analyses were performed using Stata statistical software, version 14.0 (StataCorp, College Station, TX, USA). Twosided $P<0.05$ was considered significant.

\section{Results}

\section{Demographic and clinical characteristics of CATS}

We summarized the demographic and clinical characteristics of all 149,026 patients with at least a 2-year follow-up time (Table 1). The median follow-up was over 7 years (88 months). Compared with the UOBC patients, the CATS patients were significantly younger (age of 18-45 years: $26.6 \%$ vs $18.4 \%, P<0.001$ ), more were black (black race: $13.5 \%$ vs $9.7 \%, P<0.001)$, they had a smaller tumor size ( $0-20 \mathrm{~mm}$ size: $68.4 \%$ vs $61.6 \%, P<0.001)$, the grade was III and undifferentiated (UR) $(43.4 \%$ vs $38.1 \%, P<0.001)$, and they had a higher HoR- rate (HoR-: $26.1 \%$ vs $20.8 \%$, $P<0.001)$. More CATS patients (72.9\%), compared to UOBC patients $(61.7 \%)$, received breast-conserving surgery. This phenomenon was explained by the smaller cancer of CATS and easier breast-conserving operation in the ATS. Compared to UOBC, CATS had a significantly higher $\mathrm{LN}$ positivity rate

Table I Baseline characteristics of patients with CATS or UOBC from the SEER database 1990-2012

\begin{tabular}{|c|c|c|c|}
\hline Characteristics & $\begin{array}{l}\text { CATS } \\
(n=2,683)\end{array}$ & $\begin{array}{l}\text { UOBC } \\
(n=146,343)\end{array}$ & $P$-value \\
\hline Year, \% & & & $<0.001$ \\
\hline 1990-1997 & I5. I & 12.3 & \\
\hline $1998-2005$ & 42.8 & 39 & \\
\hline $2006-2012$ & 42.1 & 48.7 & \\
\hline Age (years), \% & & & $<0.001$ \\
\hline $18-45$ & 26.6 & 18.4 & \\
\hline $46-55$ & 27.9 & 28.1 & \\
\hline $56-65$ & 22.4 & 26.8 & \\
\hline $66-79$ & 23.1 & 26.7 & \\
\hline Race, \% & & & $<0.00$ I \\
\hline White & 80.3 & 81.9 & \\
\hline Black & 13.5 & 9.7 & \\
\hline Others & 5.8 & 8.1 & \\
\hline Unknown & 0.5 & 0.4 & \\
\hline Marital status, \% & & & 0.008 \\
\hline Married & 63.9 & 61.1 & \\
\hline Not married & 33 & 35.9 & \\
\hline Unknown & 3.2 & 3.1 & \\
\hline
\end{tabular}

Table I (Continued)

\begin{tabular}{|c|c|c|c|}
\hline Characteristics & $\begin{array}{l}\text { CATS } \\
(n=2,683)\end{array}$ & $\begin{array}{l}\text { UOBC } \\
(n=\mid 46,343)\end{array}$ & $P$-value \\
\hline Laterality, \% & & & 0.902 \\
\hline Left & 49.8 & 49.7 & \\
\hline Right & 50.2 & 50.3 & \\
\hline Histology, \% & & & 0.024 \\
\hline IDC & 78.9 & 77.8 & \\
\hline ILC & 5.1 & 6.4 & \\
\hline Others & 16 & 15.8 & \\
\hline Grade, \% & & & $<0.001$ \\
\hline I & 19.4 & 20.6 & \\
\hline ॥ & 37.2 & 41.4 & \\
\hline III and UD & 43.4 & 38.1 & \\
\hline Stage, \% & & & 0.062 \\
\hline I & 47.5 & 46.8 & \\
\hline II & 37.6 & 39.5 & \\
\hline III & 15 & 13.7 & \\
\hline Tumor size, \% & & & $<0.001$ \\
\hline $0-20 \mathrm{~mm}$ & 68.4 & 61.6 & \\
\hline $21-50 \mathrm{~mm}$ & 28 & 33 & \\
\hline$>50 \mathrm{~mm}$ & 3.6 & 5.4 & \\
\hline LN status, \% & & & $<0.001$ \\
\hline Negative & 60 & 63.3 & \\
\hline Positive & 40 & 36.7 & \\
\hline LN examined, \% & & & $<0.001$ \\
\hline $1-3$ & 24 & 31.6 & \\
\hline 4-9 & 23.8 & 22.6 & \\
\hline$>9$ & 48.9 & 42.7 & \\
\hline Unknown/no examine & 3.4 & 3.2 & \\
\hline LN positive, $\%$ & & & 0.001 \\
\hline 0 & 58 & 61.7 & \\
\hline $1-3$ & 25.8 & 24.1 & \\
\hline $4-9$ & 8.5 & 7.9 & \\
\hline$>9$ & 4.4 & 3.6 & \\
\hline Unknown/no examine & 3.4 & 2.8 & \\
\hline LNR, \% & & & 0.06 \\
\hline$\leq 0.3$ & 81.5 & 83 & \\
\hline$>0.3$ and $\leq 0.7$ & 9.4 & 9.2 & \\
\hline$>0.7$ & 5.5 & 4.5 & \\
\hline Unknown/no examine & 3.6 & 3.3 & \\
\hline HoR, \% & & & $<0.001$ \\
\hline Negative & 26.1 & 20.8 & \\
\hline Positive & 73.9 & 79.2 & \\
\hline Surgery, \% & & & $<0.001$ \\
\hline Lumpectomy & 72.9 & 61.7 & \\
\hline Mastectomy & 27.1 & 38.3 & \\
\hline Radiation, \% & & & $<0.001$ \\
\hline Yes & 63.6 & 59 & \\
\hline No & 1.2 & 0.9 & \\
\hline Unknown & 35.2 & 40.1 & \\
\hline Chemotherapy, \% & & & 0.006 \\
\hline Yes & 51 & 48.4 & \\
\hline No/unknown & 49 & 51.6 & \\
\hline
\end{tabular}


(LN+: $40 \%$ vs $36.7 \%, P<0.001$ ). CATS patients received more LN examinations and had more metastatic LNs. Previous research reveals that the lymph node ratio (LNR) is a superior predictor of clinical outcome to the number of metastatic nodes. ${ }^{17,18}$ The LNR was defined as the number of positive LNs divided by the number removed. Interestingly, CATS was also associated with a higher LNR. These characteristics suggested that CATS had a distinct clinicopathological baseline from UOBC.

\section{Comparison of survival between CATS and UOBC}

Kaplan-Meier plots were utilized to evaluate the BCSS and OS in CATS and UOBC (Figure 1). Patients with CATS had a worse survival than the UOBC population for BCSS (competing risks analysis $P=0.001)$. CATS had a worse 10 -year BCSS rate over UOBC (85.1\% vs $87.3 \%$ ). There was no statistical significance in the 10-year OS between CATS and UOBC ( $78 \%$ vs $78.8 \%$, log-rank test $P=0.161$ ).

Because of the distinct clinicopathological baseline of CATS, the univariate and multivariate Cox proportional hazards model was used to balance the effect of other factors. The factors that were significantly associated with BCSS in the univariate analysis (Table S1) were included in the multivariate analysis (Table 2), such as age, year of diagnosis, race, marital status, the location of ATS or UOB, histologic type, tumor grade, tumor size, LN status, HoR status, surgery type, radiation, and chemotherapy. After adjusting for other factors, the tumor location of ATS was confirmed as an independent risk factor over UOB in both BCSS and OS (BCSS: $\mathrm{HR}=1.20, P=0.001,95 \%$ CI: 1.07-1.34; OS: HR $=1.11, P=0.019$, 95\% CI: $1.02-1.22)$.

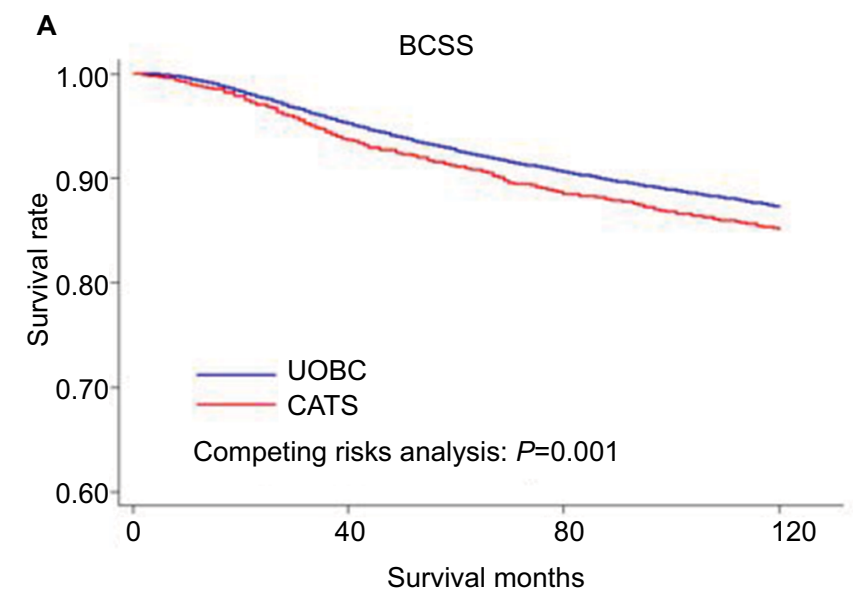

\section{Survival analysis in the matched group}

Furthermore, a 1:1 (CATS/UOBC) matched case-control analysis, by PSM, was carried out to balance the baseline differences. In total, 5,170 patients were obtained with known marital status and race information, including 2,585 patients for each location type. Ten factors were included in the PSM, including age, year of diagnosis, race, marital status, histologic type, tumor grade, tumor size, LN status, HoR status, and surgery type. No statistically significant differences were found in the characteristics between the two groups (Table S2). The survival analysis of the matched groups, consistent with the entire cohort analysis, showed that CATS presented a worse outcome for the BCSS (10-year BCSS: $85 \%$ vs $87.4 \%$, competing risks analysis $P=0.019$ ) (Figure 2) and no statistical significance for the OS (10-year OS: $78.2 \%$ vs $79.8 \%$, log-rank test $P=0.210$ ) ( $\underline{\text { Figure } S 2}$ ).

\section{Subgroup analysis and forest plot}

The above Kaplan-Meier plots and Cox analysis indicated that CATS had a worse BCSS. A subgroup survival analysis was conducted to look into the specific subgroups. HoR is one important therapeutic target in clinical practice. LN status is a key factor for American Joint Committee on Cancer stage and surgery extension, and infiltrating duct carcinoma (IDC) is the most common histology type of breast cancer. We conducted survival analysis in these five subgroups (HoR+, HoR-, LN+, LN-, and IDC). The Kaplan-Meier plots showed that the CATS patients had a diminished BCSS in the three subgroups of $\mathrm{HoR}-, \mathrm{LN}+$ and IDC (respectively, competing risks analysis: $P=0.001 ; P=0.014$; and $P=0.001$ ) and had a diminished OS in the HoR-subgroup (log-rank test $P=0.004)$ (Figure S3).

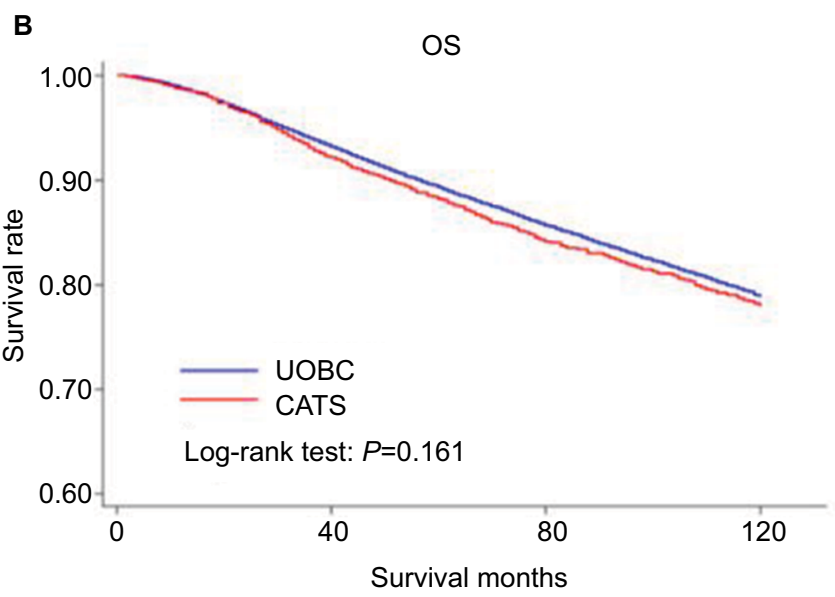

Figure I Kaplan-Meier plots of the 10-year BCSS and OS were utilized to compare CATS and UOBC.

Notes: Patients with CATS had a worse survival than the UOBC population for BCSS (competing risks analysis $P=0.00 \mathrm{I})(\mathbf{A})$. There was no statistical significance in the 10-year OS between CATS and UOBC (78\% vs 78.8\%, log-rank test $P=0.161)$ (B).

Abbreviations: BCSS, breast cancer-specific survival; CATS, carcinoma of the axillary tail of Spence; OS, overall survival; UOBC, upper outer quadrant breast cancer. 
Table 2 Multivariate Cox regression model analysis of the BCSS and OS between CATS and UOBC

\begin{tabular}{|c|c|c|c|c|c|c|}
\hline \multirow[t]{2}{*}{ Variables $^{a}$} & \multicolumn{3}{|l|}{ BCSS } & \multicolumn{3}{|l|}{ OS } \\
\hline & HR & $95 \% \mathrm{Cl}$ & $P$-values & HR & $95 \% \mathrm{Cl}$ & $P$-values \\
\hline \multicolumn{7}{|l|}{ Year } \\
\hline 1990-1997 & Reference & & & Reference & & \\
\hline 1998-2005 & 0.65 & $0.63-0.68$ & $<0.001$ & 0.77 & $0.74-0.79$ & $<0.001$ \\
\hline 2006-2012 & 0.49 & $0.47-0.52$ & $<0.001$ & 0.6 & $0.58-0.62$ & $<0.001$ \\
\hline \multicolumn{7}{|l|}{ Age (years) } \\
\hline $18-45$ & Reference & & & Reference & & \\
\hline $46-55$ & 0.96 & $0.92-1.01$ & 0.091 & 0.97 & $0.93-1.01$ & 0.191 \\
\hline $56-65$ & 1.09 & I.04-I.I5 & $<0.001$ & 1.3 & $1.25-1.36$ & $<0.001$ \\
\hline $66-79$ & 1.50 & $1.43-1.57$ & $<0.001$ & 2.69 & $2.59-2.80$ & $<0.001$ \\
\hline \multicolumn{7}{|l|}{ Race } \\
\hline White & Reference & & & Reference & & \\
\hline Black & 1.38 & $1.32-1.45$ & $<0.001$ & 1.37 & $1.32-1.42$ & $<0.001$ \\
\hline Others & 0.88 & $0.82-0.93$ & $<0.001$ & 0.81 & $0.77-0.85$ & $<0.001$ \\
\hline \multicolumn{7}{|l|}{ Marital status } \\
\hline Married & Reference & & & Reference & & \\
\hline Not married & 1.15 & $1.12-1.20$ & $<0.001$ & 1.31 & I.28-1.34 & $<0.001$ \\
\hline \multicolumn{7}{|l|}{ Location } \\
\hline UOBC & Reference & & & Reference & & \\
\hline CATS & 1.20 & I.07-1.34 & 0.001 & 1.11 & $1.02-1.22$ & 0.019 \\
\hline \multicolumn{7}{|l|}{ Histology } \\
\hline IDC & Reference & & & Reference & & \\
\hline ILC & 0.93 & $0.86-1.01$ & 0.073 & 0.85 & $0.80-0.90$ & $<0.001$ \\
\hline Others & 0.94 & $0.90-0.99$ & 0.010 & 0.92 & $0.89-0.96$ & $<0.001$ \\
\hline \multicolumn{7}{|l|}{ Grade } \\
\hline I & Reference & & & Reference & & \\
\hline II & 2.11 & $1.94-2.30$ & $<0.001$ & 1.22 & $1.17-1.28$ & $<0.001$ \\
\hline III and UD & 3.41 & $3.13-3.71$ & $<0.001$ & 1.68 & $1.60-1.76$ & $<0.001$ \\
\hline \multicolumn{7}{|l|}{ Tumor size } \\
\hline $0-20 \mathrm{~mm}$ & Reference & & & Reference & & \\
\hline $21-50 \mathrm{~mm}$ & 2.00 & $1.93-2.08$ & $<0.001$ & 1.66 & $|.6 \mathrm{I}-1.7|$ & $<0.001$ \\
\hline$>50 \mathrm{~mm}$ & 3.41 & $3.22-3.61$ & $<0.001$ & 2.79 & $2.66-2.92$ & $<0.001$ \\
\hline \multicolumn{7}{|l|}{ LN status } \\
\hline Negative & Reference & & & Reference & & \\
\hline Positive & 2.83 & $2.72-2.94$ & $<0.001$ & 2.03 & $1.97-2.09$ & $<0.001$ \\
\hline \multicolumn{7}{|l|}{ HoR } \\
\hline Negative & Reference & & & Reference & & \\
\hline Positive & 0.56 & $0.54-0.58$ & $<0.001$ & 0.64 & $0.62-0.66$ & $<0.001$ \\
\hline \multicolumn{7}{|l|}{ Surgery } \\
\hline Lumpectomy & Reference & & & Reference & & \\
\hline Mastectomy & 1.30 & $1.25-1.35$ & $<0.001$ & 1.25 & $1.21-1.29$ & $<0.001$ \\
\hline \multicolumn{7}{|l|}{ Radiation } \\
\hline Yes & Reference & & & Reference & & \\
\hline No & 2.16 & $1.90-2.45$ & $<0.001$ & 1.84 & $1.66-2.04$ & $<0.001$ \\
\hline \multicolumn{7}{|l|}{ Chemotherapy } \\
\hline Yes & Reference & & & Reference & & \\
\hline No/unknown & 0.95 & $0.91-0.99$ & 0.014 & 1.21 & $1.17-1.25$ & $<0.001$ \\
\hline
\end{tabular}

Notes: aAdjusted by Cox proportional hazards models, including location (UOBC or CATS), year of diagnosis, age, race, marital status, histology, grade, tumor size, LN status, hormone receptor status, surgery type, radiation, and chemotherapy.

Abbreviations: BCSS, breast cancer-specific survival; CATS, carcinoma of the axillary tail of Spence; HoR, hormone receptor; HR, hazard ratio; IDC, infiltrating duct carcinoma; ILC, infiltrating lobular carcinoma; LN status, lymph node status; OS, overall survival; UD, undifferentiated; UOBC, upper outer quadrant breast cancer. 


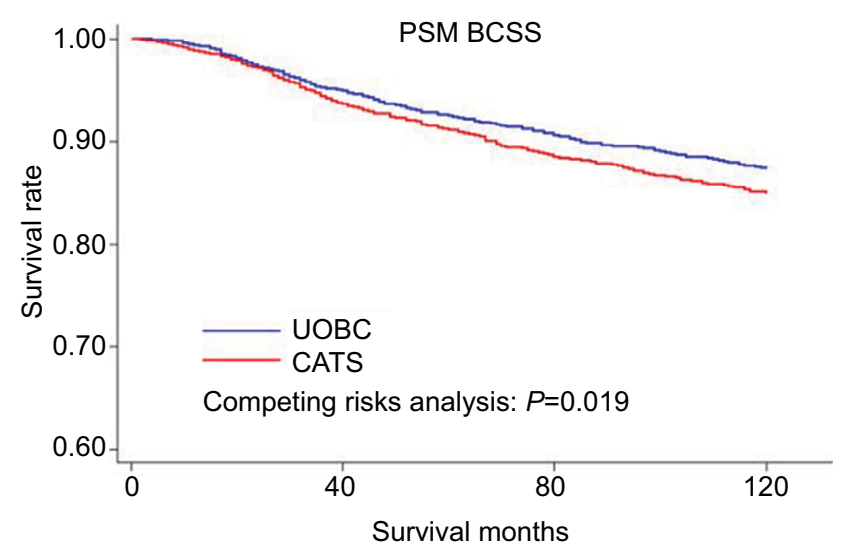

Figure 2 Kaplan-Meier plots of the 10-year BCSS comparing I:I matched CATS and $\mathrm{UOBC}$ cases.

Notes: The survival analysis of the matched groups showed that CATS presented a worse outcome for the BCSS ( 10 -year BCSS: $85 \%$ vs $87.4 \%$, competing risks analysis $P=0.019)$.

Abbreviations: BCSS, breast cancer-specific survival; CATS, carcinoma of the axillary tail of Spence; PSM, propensity score matching; UOBC, upper outer quadrant breast cancer.
Moreover, the forest plots of the HRs in the subgroups were plotted to describe the stratified prognosis value of ATS location of breast cancer (Figure 3). Briefly, the HRs of CATS versus UOBC were significant in most of the subgroups for BCSS and in almost half of the subgroups for OS. Compared with UOBC, CATS presented higher HRs for BCSS in the subgroups of diagnosis aged $66-79$ years $(\mathrm{HR}=1.46,95 \%$ CI: 1.18-1.81), IDC (HR $=1.21,95 \%$ CI: $1.07-1.37)$, Grade III and UD (HR $=1.22,95 \% \mathrm{CI}: 1.08-1.39)$, all subtypes of tumor size, $\mathrm{LN}+(\mathrm{HR}=1.17,95 \% \mathrm{CI}: 1.03-1.33)$, HoR(HR $=1.29,95 \%$ CI: 1.11-1.50), and both surgery types (lumpectomy and mastectomy, respectively, $\mathrm{HR}=1.37,95 \%$ CI: $1.18-1.58 ; \mathrm{HR}=1.33,95 \%$ CI: 1.13-1.56). Additionally, CATS was also a risk factor for OS in the subgroups of Grade III and UD (HR $=1.13,95 \%$ CI: $1.01-1.27$ ), tumor size of 21-50 $\mathrm{mm}$ and $>50 \mathrm{~mm}$ (respectively, $\mathrm{HR}=1.25,95 \% \mathrm{CI}$ : $1.09-1.43 ; \mathrm{HR}=1.48,95 \%$ CI: $1.11-1.99), \mathrm{HoR}-(\mathrm{HR}=1.22$,

B

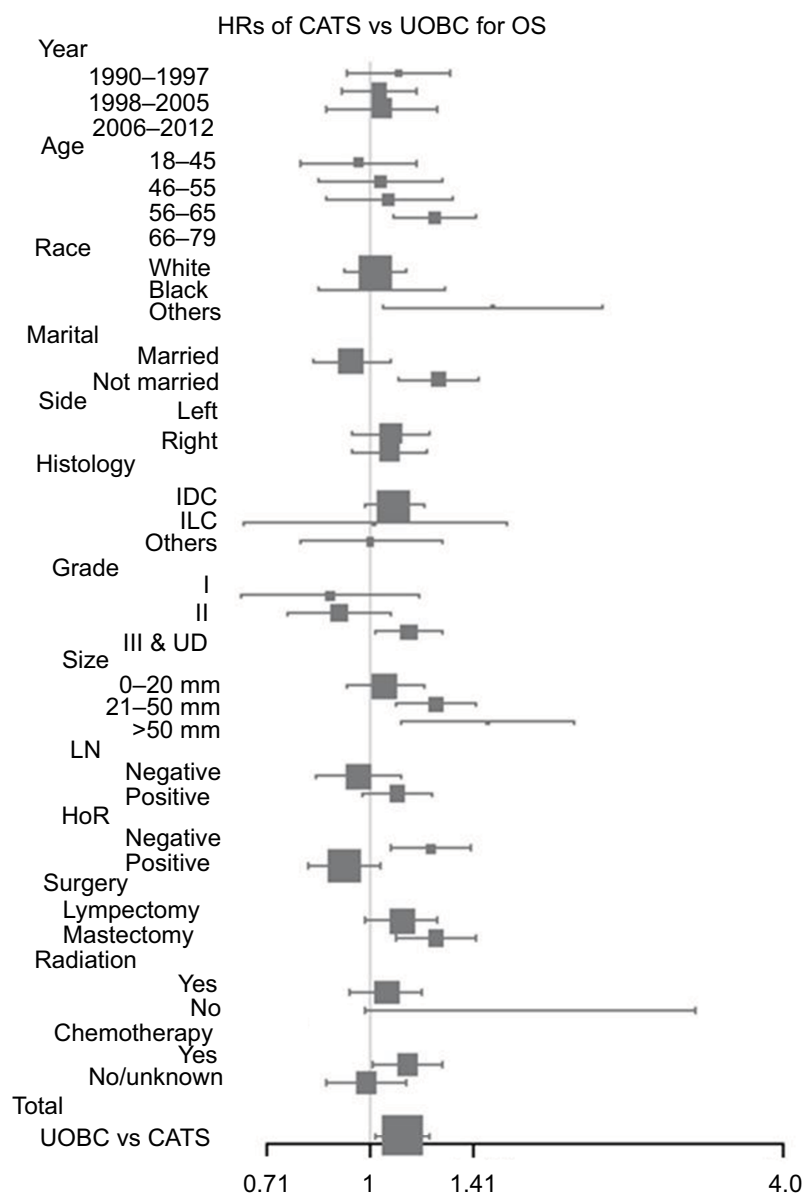

Figure 3 Forest plot of the hazard ratios for CATS versus UOBC in the subgroup analysis.

Notes: The forest plots of the HRs in the subgroups were plotted to describe the stratified prognosis value of ATS location of breast cancer. The HRs of CATS versus UOBC were significant in most of the subgroups for BCSS (A) and in almost half of the subgroups for OS (B).

Abbreviations: ATS, axillary tail of Spence; BCSS, breast cancer-specific survival; CATS, carcinoma of the axillary tail of Spence; HoR, hormone receptor; HR, hazard ratio; LN, lymph node; OS, overall survival; UD, undifferentiated; UOBC, upper outer quadrant breast cancer; IDC, infiltrating duct carcinoma; ILC, infiltrating lobular carcinoma. 
95\% CI: $1.07-1.40)$, and mastectomy ( $\mathrm{HR}=1.24,95 \% \mathrm{CI}$ : 1.08-1.43). These data suggest that some clinicopathological parameters, such as LN status, also play an important role in determining the prognosis of CATS patients.

\section{CATS related to $\mathrm{LN}$ metastasis}

We hypothesized that one possible reason for the worse prognosis of CATS is that it is "easier" to metastasize to the LN. A multivariate logistic regression analysis was utilized to assess whether CATS was the independent variable for predicting LN metastasis. Variables that were significant $(P<0.05)$ in the univariate logistic analysis (tumor location, year of diagnosis, age, race, histologic type, grade, tumor size, and HoR status) (Table S3) were included in the multivariate logistic regression analysis (Table 3 ). According to the result, CATS was more likely to metastasize to the $\mathrm{LN}$ (OR $=1.26$,

Table 3 Multivariate logistic regression analysis on LN metastasis

\begin{tabular}{|c|c|c|c|}
\hline Variables & OR & $95 \% \mathrm{Cl}$ & $P$-values \\
\hline \multicolumn{4}{|l|}{ Year } \\
\hline 1990-1997 & Reference & & \\
\hline $1998-2005$ & 0.98 & $0.95-1.02$ & 0.301 \\
\hline $2006-2012$ & 0.83 & $0.80-0.86$ & $<0.001$ \\
\hline \multicolumn{4}{|l|}{ Age } \\
\hline $18-45$ & Reference & & \\
\hline $46-55$ & 0.86 & $0.84-0.89$ & $<0.001$ \\
\hline $56-65$ & 0.74 & $0.72-0.77$ & $<0.001$ \\
\hline $66-79$ & 0.59 & $0.57-0.6 \mathrm{I}$ & $<0.001$ \\
\hline \multicolumn{4}{|l|}{ Race } \\
\hline White & Reference & & \\
\hline Black & 1.12 & $1.08-1.16$ & $<0.001$ \\
\hline Others & 0.88 & $0.84-0.92$ & $<0.001$ \\
\hline \multicolumn{4}{|l|}{ Location } \\
\hline UOBC & Reference & & \\
\hline CATS & 1.26 & $1.16-1.37$ & $<0.001$ \\
\hline \multicolumn{4}{|l|}{ Histology } \\
\hline IDC & Reference & & \\
\hline ILC & 0.92 & $0.87-0.96$ & 0.001 \\
\hline Others & 0.86 & $0.83-0.89$ & $<0.001$ \\
\hline \multicolumn{4}{|l|}{ Grade } \\
\hline I & Reference & & \\
\hline II & 1.84 & $1.78-1.91$ & $<0.001$ \\
\hline III and UD & 2.36 & $2.27-2.45$ & $<0.001$ \\
\hline \multicolumn{4}{|l|}{ Tumor size } \\
\hline $0-20 \mathrm{~mm}$ & Reference & & \\
\hline $2 \mathrm{I}-50 \mathrm{~mm}$ & 3.20 & $3.13-3.28$ & $<0.001$ \\
\hline$>50 \mathrm{~mm}$ & 8.14 & 7.7I-8.59 & $<0.001$ \\
\hline \multicolumn{4}{|l|}{ HoR } \\
\hline Negative & Reference & & \\
\hline Positive & 1.34 & $1.30-1.39$ & $<0.001$ \\
\hline
\end{tabular}

Abbreviations: BCSS, breast cancer-specific survival; CATS, carcinoma of the axillary tail of Spence; HoR, hormone receptor; IDC, infiltrating duct carcinoma; ILC, infiltrating lobular carcinoma; LN, lymph node; OR, odds ratio; OS, overall survival; UD, undifferentiated; UOBC, upper outer quadrant breast cancer.
$P<0.001,95 \% \mathrm{CI}: 1.16-1.37)$. This result confirmed the linkage of CATS and LN metastasis and partially explained the worse outcome of CATS.

\section{Discussion}

CATS has a unique anatomical position, and its clinicopathological and survival characteristics are not well investigated. We utilized 149,026 patients (including 2,683 CATS cases) from 1990 to 2012, and the median follow-up was 88 months. As the largest analysis of CATS to date, this research showed that CATS, compared to UOBC, has distinct clinicopathological characteristics and a worse prognosis.

Previous research reveals that most CATS patients are in the older age group ( $>45$ years) ${ }^{8}$ In line with previous study, our study showed that most of the CATS patients (73.4\%) were older than 45 years. In contrast with UOBC, we found that CATS is more likely to occur with younger age (Table 1). The survival analysis indicated that CATS was associated with a better BCSS than UOBC (Figure 1). After adjusting for other factors, patients with CATS have $20 \%$ more risk of dying as the result of breast cancer and have a worse OS compared to UOBC (Table 2). Kroman et $\mathrm{al}^{19}$ found that the risk of dying increased significantly (up to $21 \%$ ) with the increasing distance of the tumor location from the axilla. Based on our research, Kroman et al's ${ }^{19}$ conclusion does not apply to CATS.

Two points may explain the inferior outcomes of CATS. The first point is the understaging of CATS at diagnosis. In screening and imaging, CATS may be hidden by the pectoralis muscles or the plastic reconstruction, and thus CATS may present at a later stage. ${ }^{10}$ Additionally, the boundary between the axilla and ATS is arbitrary, and the missing potentially positive nodes in ATS may result in understaging and, hence, the undertreatment of breast cancer. The second point is the "easier" LN metastasis of CATS compared to UOBC. We conducted a multivariate logistic regression analysis, which indicated that CATS is an independent indicator for LN metastasis (Table 3). These two points have some internal relationship, in which the cause or effect is hard to determine.

This research has potential implications for clinical practice. In screening, the examination of the axilla should be stressed. The American College of Radiology Breast Imaging Reporting and Data System (BI-RADS) atlas suggested that the axilla could be scanned as well the breast parenchyma. ${ }^{20,21}$ For the suspicious finding of the axilla in screening, Okubo et $\mathrm{al}^{9}$ suggested that magnetic resonance imaging (MRI) and positron emission tomography could aid in the preoperative diagnosis of CATS, although the utility of positron emission 
tomography in the suspected breast cancer patients has not been established due to the high cost and radiation. MRI is a more practical imaging method for the accurate diagnosis of CATS. The accuracy and sensitivity of MRI were confirmed in occult breast cancer. ${ }^{22}$ The concerns of extensive examination on the axilla would be of low economic benefit and have a high false-positive rate. In treatment, we suggested the same treatment for CATS as with anatomic breast cancer of a parallel disease stage. According to our research, the survival difference between CATS and UOBC is statistically significant but slight (10-year BCSS: $85.1 \%$ vs $87.3 \%$, competing risks analysis $P=0.001)$. Surgery should be the mainstay treatment in CATS. The surgical management is left to the judgment of the surgeon. The concerning point is the surgery edge of CATS because it is difficult to identify the edge of ATS tissue. ${ }^{23}$ Significantly, it is worth noting that the subgroup analyses revealed an inferior BCSS for CATS in the LN+ subgroup (Figures S3 and 3). This result indicated that CATS patients with an $\mathrm{LN}+$ status should get more attention.

Inevitably, our research has several limitations. First, due to the continuity of UOB and ATS, the differential diagnosis of UOBC and CATS might be difficult and sometimes inaccurate, such as the case reported by Okubo et al. ${ }^{9}$ Cases with a more accurate diagnosis will support more convincing results. Second, we found a significant difference between CATS and UOBC based on the large dataset. However, the actual clinical difference may be small. SEER, as a large database, may have a risk of coding error, but it underwent quality monitoring and integrity evaluation. Third, as for treatment, the subjects with "no/unknown" radiation or chemotherapy were not guaranteed as not receiving these treatments or just missing the information. Therefore, the SEER did not help to compare the outcome by treatment received. Fourth, some important variables are missing in the SEER database, including detailed treatment, Her2 status (available since 2010), disease-free survival information, etc. Therefore, further studies are needed to investigate the molecular features of CATS to reveal its biological nature.

\section{Conclusion}

CATS is poorly studied due to its rarity and a lack of clinical awareness. Our research, for the first time, unveiled that CATS is distinct from UOBC in terms of having different characteristics and a worse prognosis. This research demonstrated that the tumor site of axillary tail tissue is an independent prognostic factor and has potential implications for clinical practice. Consistent with the previous study, the location of breast cancer is confirmed again as a significant prognostic factor.

\section{Acknowledgments}

This work was supported by grants from the National Natural Science Foundation of China (81502278, 81572583, $81372848,81370075)$, the Training Plan of Excellent Talents in Shanghai Municipality Health System (2017YQ038), the Training Plan of Excellent Talents of Fudan University Shanghai Cancer Center (YJYQ201602), the Municipal Project for Developing Emerging and Frontier Technology in Shanghai Hospitals (SHDC12010116), the Cooperation Project of Conquering Major Diseases in Shanghai Municipality Health System (2013ZYJB0302), the Innovation Team of Ministry of Education (IRT1223), and the Shanghai Key Laboratory of Breast Cancer (12DZ2260100). The funders had no role in the study design, data collection and analysis, decision to publish, or preparation of the manuscript.

\section{Disclosure}

The authors report no conflicts of interest in this work.

\section{References}

1. Colzani E, Liljegren A, Johansson AL, et al. Prognosis of patients with breast cancer: causes of death and effects of time since diagnosis, age, and tumor characteristics. J Clin Oncol. 2011;29(30):4014-4021.

2. Wo JY, Chen K, Neville BA, Lin NU, Punglia RS. Effect of very small tumor size on cancer-specific mortality in node-positive breast cancer. J Clin Oncol. 2011;29(19):2619-2627.

3. Goldhirsch A, Ingle JN, Gelber RD, et al. Thresholds for therapies: highlights of the St Gallen International Expert Consensus on the primary therapy of early breast cancer 2009. Ann Oncol. 2009;20(8):1319-1329.

4. Gaffney DK, Tsodikov A, Wiggins CL. Diminished survival in patients with inner versus outer quadrant breast cancers. J Clin Oncol. 2003;21(3):467-472.

5. Shahar KH, Buchholz TA, Delpassand E, et al. Lower and central tumor location correlates with lymphoscintigraphy drainage to the internal mammary lymph nodes in breast carcinoma. Cancer. 2005;103(7):1323-1329.

6. Colleoni M, Zahrieh D, Gelber RD, et al. Site of primary tumor has a prognostic role in operable breast cancer: the international breast cancer study group experience. J Clin Oncol. 2005;23(7):1390-1400.

7. Sohn VY, Arthurs ZM, Sebesta JA, Brown TA. Primary tumor location impacts breast cancer survival. Am J Surg. 2008;195(5):641-644.

8. Ampil F, Caldito G, Henderson B, et al. Carcinoma of the axillary tail of Spence: a case series. Anticancer Res. 2012;32(9):4057-4059.

9. Okubo M, Tada K, Niwa T, et al. A case of breast cancer in the axillary tail of Spence - enhanced magnetic resonance imaging and positron emission tomography for diagnostic differentiation and preoperative treatment decision. World J Surg Oncol. 2013;11:217.

10. Memon S, Emanuel JC. The axillary tail - an important caveat in prophylactic mastectomy. Breast J. 2008;14(3):313-314.

11. Sarp S, Fioretta G, Verkooijen HM, et al. Tumor location of the lowerinner quadrant is associated with an impaired survival for women with early-stage breast cancer. Ann Surg Oncol. 2007;14(3):1031-1039.

12. Perkins CI, Hotes J, Kohler BA, Howe HL. Association between breast cancer laterality and tumor location, United States, 1994-1998. Cancer Causes Control. 2004;15(7):637-645. 
13. Bao J, Yu KD, Jiang YZ, Shao ZM, Di GH. The effect of laterality and primary tumor site on cancer-specific mortality in breast cancer: a SEER population-based study. PLoS One. 2014;9(4):e94815.

14. Hammond MEH, Hayes DF, Dowsett M, et al. American Society of Clinical Oncology/College of American Pathologists guideline recommendations for immunohistochemical testing of estrogen and progesterone receptors in breast cancer. J Clin Oncol. 2010;28(16):2784-2795.

15. Allred DC, Carlson RW, Berry DA, et al. NCCN Task Force Report: estrogen receptor and progesterone receptor testing in breast cancer by immunohistochemistry. J Natl Compr Canc Netw. 2009;7(Suppl 6):S1-S21.

16. National Comprehensive Cancer Network. Breast Cancer (Version 3.2017). Available from: https://www.nccn.org/professionals/physician_gls/PDF/breast.pdf. Published 2017. Accessed January 7, 2018.

17. Woodward WA, Vinh-Hung V, Ueno NT, et al. Prognostic value of nodal ratios in node-positive breast cancer. J Clin Oncol. 2006;24(18):2910-2916.
18. Vinh-Hung V, Verkooijen HM, Fioretta G, et al. Lymph Node Ratio as an Alternative to $\mathrm{pN}$ Staging in Node-Positive Breast Cancer. J Clin Oncol. 2009;27(7):1062-1068.

19. Kroman N, Wohlfahrt J, Mouridsen HT, Melbye M. Influence of tumor location on breast cancer prognosis. Int J Cancer. 2003;105(4):542-545.

20. Mendelson EB, Böhm-Vélez M, Berg WA, et al. Breast Imaging Reporting and Data System, BI-RADS - Ultrasound. 2nd ed. Reston, VA: Am Coll Radiol; 2013.

21. Ferré R, AlSharif S, Paré M, Kao E, Mesurolle B. Should the axilla be included in screening US? Radiology. 2015;274(2):623-624.

22. de Bresser J, de Vos B, van der Ent F, Hulsewe K. Breast MRI in clinically and mammographically occult breast cancer presenting with an axillary metastasis: a systematic review. Eur J Surg Oncol. 2010;36(2):114-119.

23. Temple WJ, Lindsay RL, Magi E, Urbanski SJ. Technical considerations for prophylactic mastectomy in patients at high risk for breast cancer. Am J Surg. 1991;161(4):413-415.
Cancer Management and Research

\section{Publish your work in this journal}

Cancer Management and Research is an international, peer-reviewed open access journal focusing on cancer research and the optimal use of preventative and integrated treatment interventions to achieve improved outcomes, enhanced survival and quality of life for the cancer patient The manuscript management system is completely online and includes

\section{Dovepress}

a very quick and fair peer-review system, which is all easy to use. Visit $\mathrm{http}: / /$ www.dovepress.com/testimonials.php to read real quotes from published authors. 